\title{
Depósitos epitermales de alta y baja sulfuración: una tabla comparativa
}

\author{
Antoni Camprubí, Eduardo González-Partida, Gilles Levresse \\ Jordi Tritlla y Alejandro Carrillo-Chávez
}

Programa de Geofluidos, Centro de Geociencias, Universidad Nacional Autónoma de México, Campus UNAM-Juriquilla, C.P. 76230, Santiago de Querétaro, Qro.

*camprubi@geociencias.unam.mx

\section{Introducción}

El presente trabajo es un compendio actualizado, en forma de tabla comparativa, de las características distintivas y analogías entre los dos tipos de depósitos epitermales, denominados según la terminología actualmente más empleada: alta (AS) y baja sulfuración (BS), según Hedenquist (1987). Este compendio está basado en los previamente realizados por Hayba et al. (1985), White y Hedenquist (1990), Sillitoe (1993), White et al. (1995), y completado principalmente a partir de Heald et al. (1987), Hedenquist y Lowenstern (1994), Arribas (1995), Simmons (1995), Hedenquist (1996) y Hedenquist et al. (2000). El orden de la tabla está dispuesto como "aproximación gradual” a los tipos de depósitos, esto es, desde la definición de los tipos de rocas genéticamente asociados, pasando por el reconocimiento de las evidencias de depósitos epitermales, hasta sus características más en detalle y los datos resultantes de su investigación geoquímica. De esta forma, se pretende ofrecer un orden lógico de reconocimiento y diferenciación desde el ámbito de la exploración hasta el estudio genético de los mismos. Finalmente, se hace referencia a diversos ejemplos de cada tipo, intentando cubrir al máximo su diversidad espacial, temporal, de estilo de mineralización, etc. 


\section{Tabla comparativa de las características de los depósitos epitermales de alta y baja sulfuración}

\subsection{Reconocimiento macro- a mesoscópico}

Epitermales de alta sulfuración (AS) Epitermales de baja sulfuración (BS)

Rocas volcánicas relacionadas

Controles de emplazamiento

Extensión de la zona de alteración periférica

Alteración asociada

(ver Figura 1)

Minerales clave de alteración
proximal

Geometría del cuerpo mineralizado

Características distintivas o notables

Carácter de la mineralización económica
Vulcanismo subaéreo, rocas ácidas a intermedias (esencialmente andesitariodacita).

Encajante de cualquier tipo.

Fallas a escala regional o intrusiones subvolcánicas ${ }^{(1)}$.

Área extensa (comúnmente varios $\mathrm{km}^{2}$ ) y visualmente prominente.

Extensa alteración propilítica en zonas adyacentes con baja relación agua/roca. Depósitos profundos: intensa alteración pirofilita-mica blanca. Depósitos someros: núcleo de sílice masiva, con un estrecho margen de alunita y caolinita que hacia el exterior es de mica blanca y arcillas interestratificadas. Depósitos subsuperficiales: ingente alteración argílica.

Clorita: raramente.

Generalización: alt. argílica avanzada $\rightarrow$ argílica ( \pm sericítica).

Alunita cristalina; en profundidad, pirofilita.

\section{Relativamente pequeño y} equidimensional.

Presencia de niveles calentados por vapor (niveles superficiales), cuarzo poroso o vuggy (niveles intermedios), mineralización superpuesta a depósitos porfíricos (en profundidad).

Típicamente diseminada, tanto en zona de mica blanca-pirofilita como en sílice masiva. Poco común como relleno de cavidades o porosidad. La mineralización está asociada habitualmente a alteración argílica avanzada, típicamente con abundante pirita.
Vulcanismo subaéreo, rocas ácidas a intermedias (andesita-riodacitariolita). Encajante de cualquier tipo.

Cualesquiera fallas o zonas de fractura estrechamente relacionadas a centros volcánicos.

Generalmente bastante restringida y de visualización muy sutil, aunque puede abarcar áreas relativamente extensas.

Extensa alteración propilítica en zonas adyacentes con baja relación agua/roca. Gran cantidad de mica blanca en zonas con alta relación agua/roca. Alteración argílica dominante conforme disminuye la temperatura. Los gases escapados a partir de ebullición pueden originar alteración argílica o argílica avanzada en la periferia, o bien superpuesta a partir de fluidos profundos.

Clorita: común.

Generalización: alt. sericítica $\rightarrow$ argílica.

Sericita o illita \pm adularia; roscoelita (mica-V) en depósitos asociados a rocas alcalinas; a veces, clorita.

Tamaño variable y morfología tabular.

Presencia de sínteres y niveles o cobijaduras de calcedonia (niveles superficiales), posible presencia de vetas de sulfuración intermedia en inmediaciones de cuerpos de alta sulfuración (en profundidad).

Característicamente como relleno de cavidades o porosidad, en vetas con contactos netos con el encajante. Típico relleno de filones en bandas, comúnmente con brechificaciones polifásicas. Hacia la superfície se presenta en stockwork o diseminada, según la naturaleza de la permeabilidad primaria y secundaria local. 
2.2. Reconocimiento meso- a microscópico

Epitermales de alta sulfuración (AS) Epitermales de baja sulfuración (BS)

Ganga de cuarzo

Otros minerales de la ganga

Abundancia de sulfuros

Minerales metálicos clave
De grano fino, masivo, originado principalmente por reemplazamiento; el cuarzo es residual (vuggy).

Calcedonia: ausente en la mayoría.

Carbonatos: ausentes.

Adularia: ausente.

Alunita y pirofilita: pueden ser abundantes.

Barita: diseminada con la mena.

Azufre nativo: suele estar presente, rellenando cavidades.

Caolinita.

10-90\% del volumen total, sobre todo de grano fino, pirita con textura parcialmente laminada.

Contenido de azufre total típicamente alto. El contenido en metales base puede ser alto $(\mathrm{Cu})$.

Pirita, enargita-luzonita, calcopirita, calcosita, covellita, bornita, tetraedrita-tenantita, oro (esfalerita, galena, telururos).

Arsenopirita: poco común.

Sulfosales de Ag: raramente.

Seleniuros: prácticamente ausentes.

Bismutinita: ocasionalmente.
Cuarzo y calcedonia dispuestos en todo tipo de texturas primarias, de recristalización o reemplazamiento de carbonatos $^{(2)}$.

Calcedonia: común, en bandas.

Carbonatos: presentes, comúnmente calcita y rodocrosita.

Adularia: diseminada y en vetas.

Alunita y pirofilita: escasas.

Barita y/o fluorita: presentes localmente; la barita se halla por lo común por encima de la mena.

Azufre nativo: ausente.

Illita.

1-20\% del volumen total, pero típicamente menos del $5 \%$, pirita predominante.

Contenido de azufre total típicamente bajo. Bajo contenido en metales base $(\mathrm{Pb}, \mathrm{Zn})$, aunque en numerosos depósitos son relativamente abundantes.

Pirita, esfalerita, marcasita, galena, electrum, oro (sulfosales de Ag, arsenopirita, argentita, calcopirita, tetraedrita).

Telururos: relativamente abundantes en algunos depósitos ${ }^{(3)}$.

Enargita: muy raramente.

Seleniuros: poco comunes.

Bismutinita: muy raramente ${ }^{(4)}$. 
2.3. Datos analíticos

Epitermales de alta sulfuración (AS) Epitermales de baja sulfuración (BS)

Profundidad de formación

Rango de temperaturas de formación

Carácter de los fluidos

(ver Figura 1)

Edad

Diferencia de edad entre las rocas igneas genéticament asociadas y la mineralización

Distancia lateral de formación desde el foco de calor

Origen del azufre

Origen del plomo

Metales predominantes

Metales presentes localmente
En su mayor parte, entre 500 (?) y 2,000 (?) m bajo la paleosuperfície.

Generalmente, entre $100-320^{\circ} \mathrm{C}$ (la mayoría entre $170-320^{\circ} \mathrm{C}$; en ocasiones, hasta $\left.480^{\circ} \mathrm{C}^{(5)}\right)$.

En su mayor parte, de baja salinidad (1-24\% en peso de $\mathrm{NaCl}$ eq.); algunos, de alta salinidad (hasta casi 50\% en peso de $\mathrm{NaCl}$ eq. ${ }^{(5)}$ ).

Magmáticos; mezcla con aguas meteóricas. Pueden evolucionar desde un fluido temprano muy reactivo, que lixivia la roca, hasta otro más reducido, que puede originar la mineralización.

Oxidados.

$\mathrm{pH}$ ácido debido a $\mathrm{H}_{2} \mathrm{~S}$ y $\mathrm{HCl}$ magmá-tico, neutralizado al reaccionar con el encajante; dilución.

Por lo general, Terciaria o más reciente. Ejemplos hasta el Neoproterozoico y el Arqueano(7).

Por lo general, entre 0.3 y 2 M.a.

Aproximadamente sobre la vertical del foco de calor motor del hidrotermalismo.

Profundo, probablemente magmático.

Rocas volcánicas o fluidos magmáticos.

$\mathrm{Cu}, \mathrm{Au}, \mathrm{As}(\mathrm{Ag}, \mathrm{Pb})$

Bi, Sb, Mo, Sn, Zn, Te (Hg)
En su mayor parte, entre 0 y $1,000 \mathrm{~m}$.

Generalmente, entre $100-320^{\circ} \mathrm{C}$ (la mayoría entre $150-250^{\circ} \mathrm{C}^{(6)}$ )

Baja salinidad $(0-15 \%$ en peso de $\mathrm{NaCl}$ eq.).

Aguas meteóricas; posible interacción con fluidos de origen magmático.

Reducidos.

$\mathrm{pH}$ aproximadamente neutro; puede volverse alcalino debido a ebullición; los gases separados pueden ser oxidados y producir fluidos ácidos.

Por lo general, Terciaria o más reciente. Ejemplos hasta el Neoproterozoico y Paleoproterozoico ${ }^{(8)}$.

Entre 1 y 10 M.a. (frecuentemente entre 2 y 4 M.a.)

Hasta varios kilómetros lateralmente, más cercanos al foco de calor en el subtipo de epitermales de sulfuración intermedia.

Profundo, probablemente debido al lixiviado del encajante en profundidad.

Rocas precámbricas o fanerozoicas en las que se emplaza el vulcanismo.

$\mathrm{Au}, \mathrm{Ag}(\mathrm{Zn}, \mathrm{Pb}, \mathrm{Cu})$

Mo, Sb, As (Te, Se, Hg) 
2.3. Datos analíticos (continuación)

Epitermales de alta sulfuración (AS) Epitermales de baja sulfuración (BS)

Clasificaciones posibles

Manifestaciones de sistemas análogos actualísticos
Según estilo de mineralización: en diseminaciones (Chinkuashih); en filones (El Indio); en brechas (Wafi River).

Según contenido de metales base: "rico" (Motomboto); "pobre" (Nalesbitan).

Según contexto geológico: estratovolcanes andesíticos; vulcanismo tipo Cordillera (Lepanto); islas volcánicas oceánicas.

Fumarolas y fuentes termales de alta temperatura cercanas al foco volcánico.
Según estilo de mineralización: en stockwork (McLaughlin); en diseminaciones (Cracow); en vetas (Hishikari); en brechas (Kerimenge).

Según contenido de metales base: "rico" (Fresnillo); "pobre" (Tayoltita).

Según contexto geológico: depresiones con vulcanismo silícico (Ohakuri Dam, N.Z.); estratovolcanes andesíticos (Woodlark); vulcanismo tipo Cordillera (Acupan); islas volcánicas oceánicas (Ladolam).

Según profundidad de formación: "someros" (depósitos de Norteamérica); "profundos", (depósitos del SW del Pacífico).

Según la mineralogía de las asociaciones de sulfuros: "sufuración intermedia" y "baja sulfuración" (término extremo). Igualmente, se distingue el subtipo de "epitermales alcalinos", tanto en base a su mineralogía como a su asociación con rocas volcánicas alcalinas.

Sistemas geotérmicos con fuentes termales de $\mathrm{pH}$ neutro, mud pools. 
Argentina: La Mejicana y Nevados del Famatina ${ }^{(9)}$.

Australia: Temora ${ }^{(9)}$.

Bulgaria: Chelopech, Srednogorie ${ }^{(9)}$.

Chile: El Indio-Tambo ${ }^{(4,9)}$.

China: Zijinshan ${ }^{(9)}$.

Corea del Sur: Seongsan-Ogmaesan ${ }^{(9)}$.

España: Rodalquilar ${ }^{(9)}$.

EE.UU.: Goldfield, Paradise Peak, Summitville(9).

Fiji: Mt. Kasi ${ }^{(9)}$.

Filipinas: Lepanto, Nalesbitan ${ }^{(9)}$.

Indonesia: Motomboto ${ }^{(9,21)}$.

Japón: Mitsumori-Nukeishi, Nansatsu( ${ }^{(9)}$.

México: Mulatos ${ }^{(10,11)}$, El Sauzal(11).

Papúa-Nueva Guinea: Wafi River ${ }^{(9)}$.

Perú: Ccahuarso, Cerro de Pasco, Julcani, Castrovirreyna ${ }^{(4,9)}$.

Suecia: Enåsen ${ }^{(9)}$.

Taiwan: Chinkuashih(9).
Argentina: Cerro Vanguardia, Manantial Espejo ${ }^{(12)}$.

Australia: Cracow, Mount Coolon(13).

Canadá: Freegold Mountain, Toodoggone ${ }^{(14)}$.

Chile: Inca de Oro ${ }^{(15)}$.

China: Rushan ${ }^{(16)}$.

Corea del Sur: Jeongju-Buan ${ }^{(17)}$.

EE.UU.: Comstock ${ }^{(18)}$, Creede ${ }^{(19)}$, McLaughlin ${ }^{(20)}$.

Fiji: Emperor(18).

Filipinas: Antamok-Acupan ${ }^{(3,18)}$.

Indonesia: Gunung Pongkor, Kelian(21).

Japón: Hishikari(18).

Marruecos: Imiter ${ }^{(28)}$.

México: Fresnillo(18), Guanajuato(2), Tayoltita( ${ }^{(18)}$, Temascaltepec (23), Topia $^{(24)}$.

Papúa-Nueva Guinea: Ladolam, Porgera(25).

Perú: Arcata, Casapalca, Caylloma, Orcopampa $(4)$.

Rusia: Bereznjakovskoje, Julietta ${ }^{(26) .}$

Turquía: Mastra y otros ${ }^{(27)}$.

Referencias en la tabla: (1) Relacionado con la permeabilidad, con control litológico del flujo en presencia de unidades permeables; en caso contrario, existe un fuerte control estructural (Hedenquist et al., 1994). (2) Véase Dong et al. (1995). (3) Véase Cooke y Bloom (1990) y Cooke et al. (1996). (4) Véase Ericksen y Cunningham (1993). (5) Las más altas salinidades y temperaturas pueden ser debidas a una intensa etapa de alteración previa a la deposición mineral. (6) Muy raramente se sobrepasan $\operatorname{los} 350^{\circ} \mathrm{C}$, en cuyo caso se interpreta la presencia de procesos poco usuales: véase McInnes et al. (1990). (7) Huckerby et al. (1983), y Hallberg (1994). (8) Penczak y Mason (1997), y Cheilletz et al. (2002). (9) Incluidos en la relación de Arribas (1995). (10) Staude (2001). (11) Gray (2001). (12) Haller (1997), y Etcheverry et al. (1997). (13) Dong y Zhou (1996), y Wood et al. (1990). (14) Panteleyev (1988), y Thiersch et al. (1997). (15) Palacios et al. (1992). (16) Jianping et al. (1996). (17) So y Yun (1996). (18) Incluidos en la relación de Simmons (1995). (19) Hayba et al. (1985). (20) Sherlock et al. (1995). (21) Sillitoe (1994). (22) Mango et al. (1991). (23) Camprubí et al. (2001a, 2001b). (24) Loucks et al. (1988). (25) White et al. (1995). (26) Lehmann et al. (1997) y Strujkov et al. (1996). (27) Tüysüz y Akçay (1997). (28) Cheilletz et al. (2002).

Caracterización completa de las asociaciones de alteración hidrotermal en ambos tipos de depósitos epitermales, y características detalladas de ambos tipos según su profundidad de formación en Hedenquist et al. (2000). Para más ejemplos de depósitos epitermales de ambos tipos, ver Arribas (1995), White et al. (1995), y Hedenquist et al. (2000). Datos generales sobre depósitos epitermales en México en Camprubí et al. (1999 y 2003), y Albinson et al. (2001). Datos sobre paragénesis de alteraciones hidrotermales, geotermómetros minerales y características de sistemas geotérmicos actuales análogos a los depósitos epitermales en Reyes (1990 y 1991), Hedenquist et al. (1992), Simmons y Browne (1997) y González-Partida (2000). 

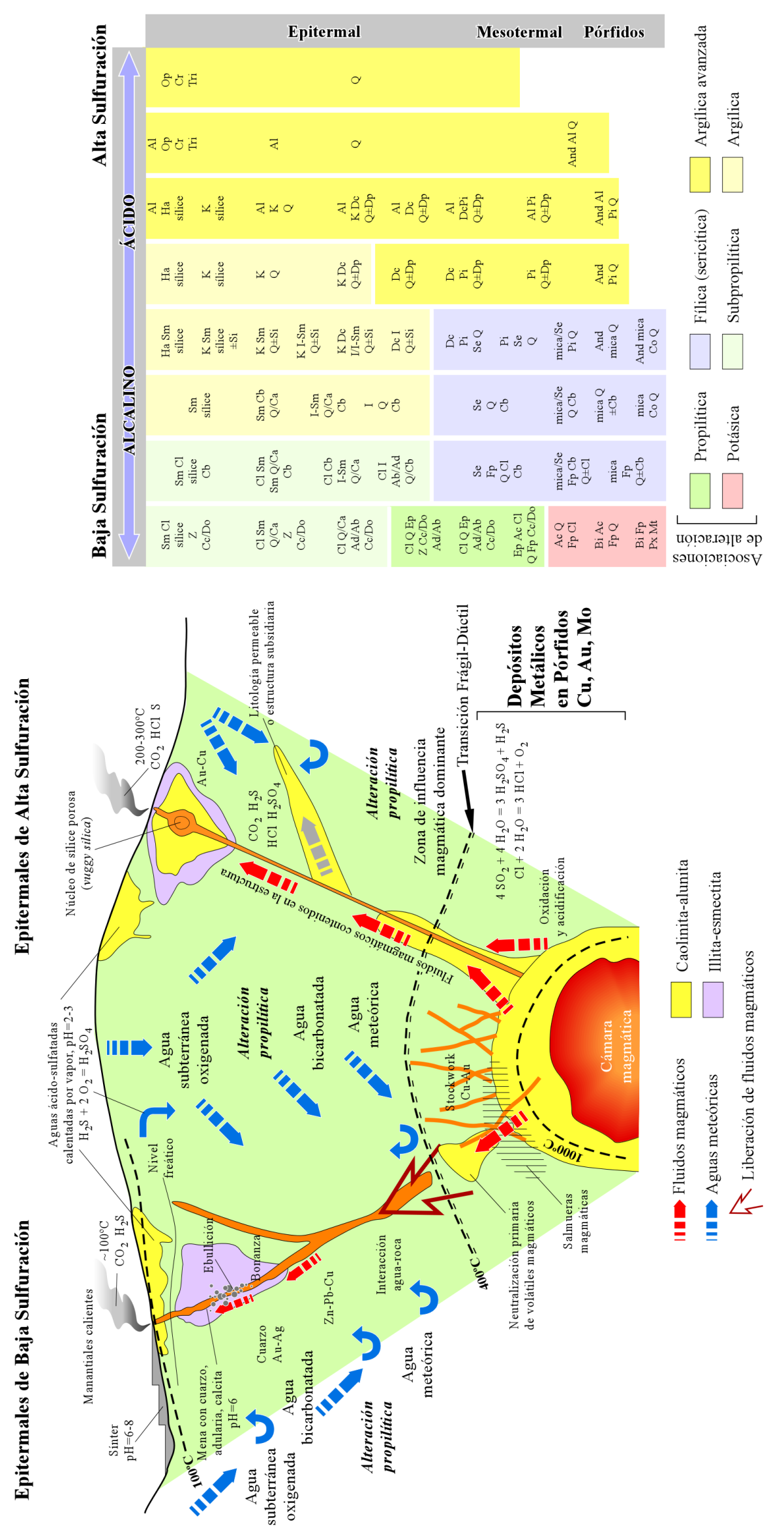

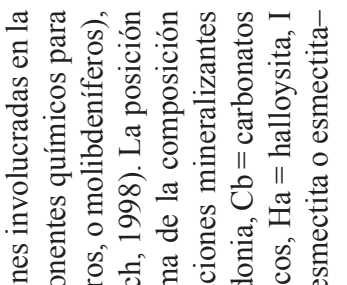

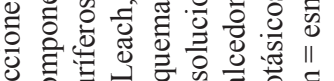

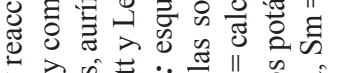

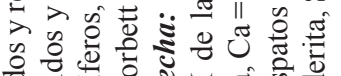

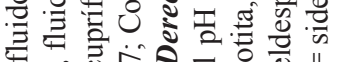

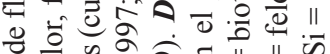

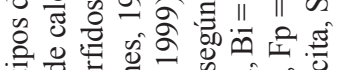
Ұ \&

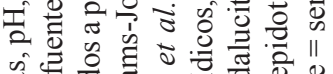

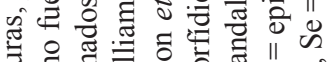

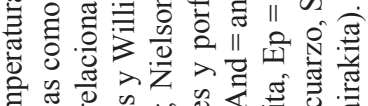

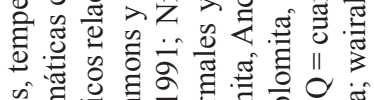

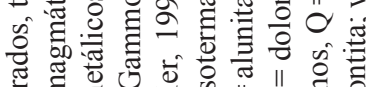

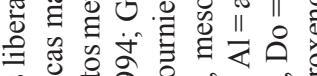

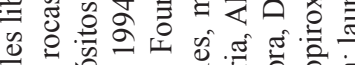
늘

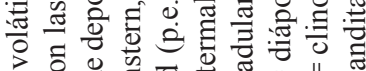

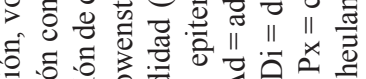

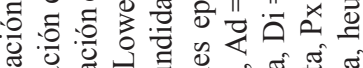

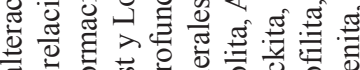
ঠ के

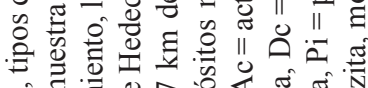

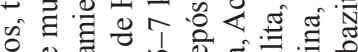

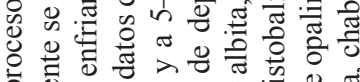
岂

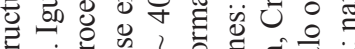

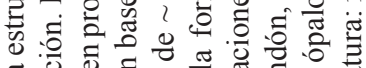

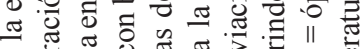

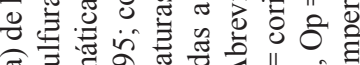

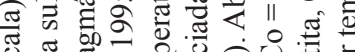

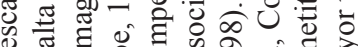

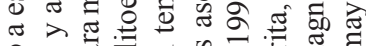

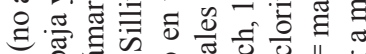

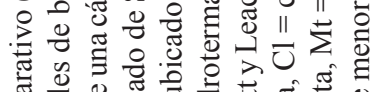

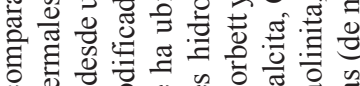
ठ.

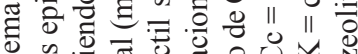

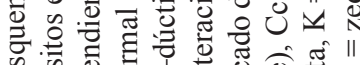
论

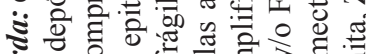

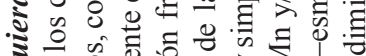

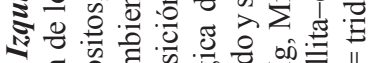

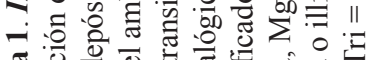
ॠ

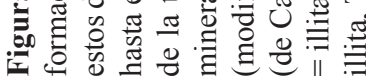




\section{Agradecimientos}

El presente trabajo se ha beneficiado de los comentarios de Carles Canet, Miguel Miranda Gasca y Ma. Teresa Orozco.

\section{Referencias bibliográficas}

Albinson, T., Norman, D.I.E, Cole, D., Chomiak, B.A., 2001, Controls on formation of low-sulfidation epithermal deposits in Mexico: constraints from fluid inclusion and stable isotope data, in Albinson, T., Nelson, C.E. (eds.), New Mines and Discoveries in Mexico and Central America: Society of Economic Geologists Special Publication Series, 8, 1-32.

Arribas, A. Jr., 1995, Characteristics of high-sulfidation epithermal deposits, and their relation to magmatic fluid, in Thompson, J.F.H. (ed.), Magmas, Fluids and Ore Deposits: Mineralogical Association of Canada, Short Course Series, 23, 419-454.

Camprubí, A., Prol-Ledesma, R.M., Tritlla, J., 1999, Comments on 'Metallogenic evolution of convergent margins: selected ore deposit models' by S.E. Kesler: Ore Geology Reviews, 14, 71-76.

Camprubí, A., Canals, À., Cardellach, E., Prol-Ledesma, R.M., Rivera, R., 2001a, The La Guitarra Ag-Au low sulfidation epithermal system, Temascaltepec district, Mexico: vein structure, mineralogy, and sulfide-sulfosalt chemistry, in Albinson, T., Nelson, C.E. (eds.), New Mines and Discoveries in Mexico and Central America: Society of Economic Geologists Special Publication Series, 8, 133-158.

Camprubí, A., Cardellach, E., Canals, À., Lucchini, R., 2001b, The La Guitarra $\mathrm{Ag}-\mathrm{Au}$ low sulfidation epithermal system, Temascaltepec district, Mexico: fluid inclusion and stable isotope data, in Albinson, T., Nelson, C.E. (eds.), New Mines and Discoveries in Mexico and Central America: Society of Economic Geologists Special Publication Series, 8, 159-185.

Camprubí, A., Ferrari, L., Cosca, M.A., Cardellach, E., Canals, À., 2003, Ages of epithermal deposits in Mexico: regional significance and links with the evolution of Tertiary volcanism: Economic Geology, en prensa.

Cheilletz, A., Levresse, G., Gasquet, D., Azizi Samir, M.R., Zyadi, R., Archibald, D., 2002, The giant Imiter silver deposit: Neoproterozoic epithermal mineralisation in the Anti-Atlas, Morocco: Mineralium Deposita, 37, 772-781.

Cooke, D.R., Bloom, M.S., 1990, Epithermal and subjacent porphyry mineralization, Acupan, Baguio District, Philippines: a fluidinclusion and paragenetic study, in Hedenquist, J.W., White, N.C., Siddeley, G. (eds.), Epithermal Gold Mineralization of the CircumPacific: Geology, Geochemistry, Origin and Exploration, I: Journal of Geochemical Exploration, 35, 297-340.

Cooke, D.R, McPhail, D.C., Bloom, M.S., 1996, Epithermal gold mineralization, Acupan, Baguio District, Philippines: geology, mineralization, alteration, and the thermochemical environment of ore deposition: Economic Geology, 91, 243-272.

Corbett, G.J., Leach, T.M., 1998, Southwest Pacific rim gold-copper systems; structure, alteration and mineralization: Society of Economic Geologists, Special Publication Series, 6, 238 p.

Dong, G., Zhou, T., 1996, Zoning in the Carboniferous-lower Permian Cracow epithermal vein system, central Queensland, Australia: Mineralium Deposita, 31, 210-224.

Dong, G., Morrison, G., Jaireth, S., 1995, Quartz textures in epithermal veins, Queensland - classification, origin, and implication: Economic Geology, 90, 1841-1856.

Ericksen, G.E., Cunningham, C.G., 1993, Epithermal precious-metal deposits hosted by the Neogene and Quaternary volcanic complex in the Central Andes, in Kirkham, R.V., Sinclair, W.D., Thorpe, R.I., Duke, J.M. (eds.), Mineral Deposit Modeling: Geological Association of Canada, Special Paper, 40, 419-431.

Etcheverry, R., Echeveste, H., Schalamuk, I., Ametrano, S., 1997, Manantial Espejo: a Jurassic epithermal gold mineralization, Santa
Cruz, Argentina, in Papunen, H. (ed.), Mineral Deposits: Research and Exploration - where do they meet?: Rotterdam-Brookfield, A.A. Balkema, 177-180.

Fournier, R.O., 1991, The transition from hydrostatic to greater than hydrostatic fluid pressure in presently active continental hydrothermal systems in crystalline rock: Geophysical Research Letters, 18, 955-958.

Gammons, C.H., Williams-Jones, A.E., 1997, Chemical mobility of gold in the porphyry-epithermal environment. Economic Geology, 92, 45-59.

González-Partida, E., 2000, Evidencias de evolución de un fluido básico a ácido a partir del análisis de la alteración hidrotermal del campo geotérmico de Los Azufres, Michoacán: Revista Mexicana de Ciencias Geológicas, 17, 76-82.

Gray, M.D., 2001, Exploration criteria for high sulfidation gold deposits in Mexico, in Corona-Esquivel, R., Gómez-Godoy, J. (eds.), XXIV Convención Nacional de la AIMMGM, Acta de Sesiones: Acapulco, México, Asociación de Ingenieros de Minas, Metalurgistas y Geólogos de México, 68-71.

Hallberg, A., 1994, The Enåsen gold deposit, central Sweden: a paleoproterozoic high-sulfidation epithermal mineralization: Mineralium Deposita, 29, 150-162.

Haller, M.J., 1997. The rhyolite field related gold deposits from Patagonia, in Papunen, H. (ed.), Mineral Deposits: Research and Exploration - where do they meet?: Rotterdam, Brookfield, A.A. Balkema, 201-203.

Hayba, D.O., Bethke, P.M., Heald, P., Foley, N.K., 1985, Geologic, mineralogic and geochemical characteristics of volcanic-hosted epithermal precious-metal deposits, in Berger, B.R., Bethke, P.M. (eds.), Geology and Geochemistry of Epithermal Systems: Littleton, Reviews in Economic Geology, 2, 129-167.

Heald, P., Foley, N.K., Hayba, D.O., 1987, Comparative anatomy of volcanic-hosted epithermal deposits: acid-sulfate and adulariasericite types: Economic Geology, 82, 1-26.

Hedenquist, J.W., 1987, Mineralization associated with volcanic-related hydrothermal systems in the Circum-Pacific Basin, in Transactions of the $4^{\text {th }}$ Circum Pacific Energy and Mineral Resources Conference, Singapore, 1986, Oklahoma, Circum Pacific Council for Energy and Mineral Resources, 1-26.

Hedenquist, J.W., 1996, Hydrothermal systems in volcanic arcs. Origin of and exploration for epithermal gold deposits: Université de Genève, Département de Minéralogie, Short course volume, $139 \mathrm{p}$.

Hedenquist, J.W., Lowenstern, J.B., 1994, The role of magmas in the formation of hydrothermal ore deposits: Nature, 370, 519-527.

Hedenquist, J.W., Reyes, A.G., Simmons, S.F., Taguchi, S., 1992, The thermal and geochemical structure of geothermal and epithermal systems: a framework for interpreting fluid inclusion data: European Journal of Mineralogy, 4, 989-1015.

Hedenquist, J.W., Matsuhisa, Y., Izawa, E., White, N.C., Giggenbach, W.F., Aoki, M., 1994, Geology, geochemistry, and origin of highsulfidation $\mathrm{Cu}-\mathrm{Au}$ mineralization in the Nansatsu District, Japan: Economic Geology, 89, 1-30.

Hedenquist, J.W., Arribas, A. Jr., Urien-Gonzalez, E., 2000, Exploration for epithermal gold deposits, in Hagemann, S.G., Brown, P.E. (eds.), Gold in 2000: Society of Economic Geologists, Reviews in Economic Geology, 13, 245-277.

Huckerby, J.A., Moore, J.McM., Davis, G.R., 1983, Tectonic control of mineralization at Mahd adh Dhahab gold mine, western Saudi Arabia: Institution of Mining and Metallurgy Transactions, 92, B171-B182

Jianping, Z., Kai, H., Jianjun, L., 1996, Genesis and geologicalgeochemical characters of the Rushan gold deposit, Shandong, China: Chinese Journal of Geochemistry, 15, 203-212.

Lehmann, B., Heinhorst, J., Weisser, D.J., Fedesejev, V., Neumann, M., 1997, Exploration for epithermal gold mineralization in the southern Urals, Russia: the Bereznjakovskoje gold deposit, in Papunen, $\mathrm{H}$. (ed.), Mineral Deposits: Research and Exploration - where do they meet?: Rotterdam, Brookfield, A.A. Balkema, 233-236.

Loucks, R.R., Lemish, J., Damon, P.E., 1988, Polymetallic fissure vein mineralization, Topia, Durango, Mexico: Part I. District geology, 
geochronology, hydrothermal alteration, and vein mineralogy: Economic Geology, 83, 1499-1528.

Mango, H., Zantop, H., Oreskes, N., 1991, A fluid inclusion and isotope study of the Rayas $\mathrm{Ag}-\mathrm{Au}-\mathrm{Cu}-\mathrm{Pb}-\mathrm{Zn}$ mine, Guanajuato, Mexico: Economic Geology, 86, 1554-1561.

McInnes, B.I, Crocket, J.H., Goodfellow, W.D., 1990, The Laforma deposit, an atypical epithermal-Au system at Freegold Mtn., Yukon Territory, Canada, in Hedenquist, J.W., White, N.C., Siddeley, G. (eds.), Epithermal Gold Mineralization of the Circum-Pacific: Geology, Geochemistry, Origin and Exploration, II: Journal of Geochemical Exploration, 36, 73-102.

Nielson, D.L., Moore, J.N., Heizler, M.T., 1999, Lower limits of hydrothermal circulation in the Tiwi geothermal fluid, Luzon, in 24th Workshop on Geothermal Reservoir Engineering, Proceedings: Standford, California, Stanford University, $7 \mathrm{p}$.

Palacios, C., Lahsen, A., Sylvester, H., 1992, Low-sulfur epithermal gold mineralization at Inca de Oro, northern Chile: mineralogy and fluid inclusions: Journal of South American Earth Sciences, 6, 183-189.

Panteleyev, A., 1988. A Canadian cordilleran model for epithermal goldsilver deposits, in Roberts, R.G., Sheahan, P.A. (eds.), Ore Deposit Models: Ontario, Geological Association of Canada, Geoscience Canada Reprint Series, 3, 31-43.

Penczak, R.S., Mason, R., 1997, Metamorphosed Archean epithermal $\mathrm{Au}-\mathrm{As}-\mathrm{Sb}-\mathrm{Zn}-(\mathrm{Hg})$ vein mineralization at the Campbell mine, Northwestern Ontario: Economic Geology, 92, 696-719.

Reyes, A.G., 1990, Petrology of Philippine geothermal systems and the application of alteration minerlogy to their assessment: Journal of Volcanology and Geothermal Research, 43, 279-309.

Reyes, A.G., 1991, Mineralogy, distribution and origin of acid alteration in Philippine geothermal systems: Geological Survey of Japan Reports, 277, 59-65.

Sherlock, R.L., Tosdal, R.M., Lehrman, N.J., Graney, J.R., Losh, S., Jowett, E.C., Kesler, S.E., 1995, Origin of the McLaughlin mine sheeted vein complex: metal zoning, fluid inclusion, and isotopic evidence: Economic Geology, 90, 2156-2181.

Sillitoe, R.H., 1993, Epithermal models: genetic types, geometrical controls and shallow features, in Kirkham, R.V., Sinclair, W.D., Thorpe, R.I., Duke, J.M. (eds.), Mineral Deposit Modeling: Toronto, Geological Association of Canada, Special Paper, 40, 403417.

Sillitoe, R.H., 1994, Indonesian mineral deposits — introductory comments, comparisons and speculations: Journal of Geochemical Exploration, 50, 1-11.

Sillitoe, R.H., 1995, The influence of magmatic-hydrothermal models on exploration strategies for volcano-plutonic arcs, in Thompson, J.F.H. (ed.), Magmas, Fluids and Ore Deposits: Ontario, Mineralogical Association of Canada, Short Course Series, 23, 511525.

Simmons, S.F., 1995, Magmatic contributions to low-sulfidation epithermal deposits, in Thompson, J.F.H. (ed.), Magmas, Fluids and Ore Deposits: Ontario, Mineralogical Association of Canada, Short Course Series, 23, 455-477.
Simmons, S.F., Browne, P.R.L., 1997, Saline fluid inclusions in sphalerite from the Broadlands-Ohaaki geothermal system: a coincidental trapping of fluids boiled towards dryness: Economic Geology, 92, 485-489.

So, C.-S., Yun, S.-T., 1996, Geochemical evidence of progressive meteoric water interaction in epithermal $\mathrm{Au}-\mathrm{Ag}$ mineralization, Jeongju-Buan District, Republic of Korea: Economic Geology, 91, 636-646.

Staude, J.-M., 2001, Geology, geochemistry, and formation of $\mathrm{Au}-(\mathrm{Cu})$ mineralization and advanced argillic alteration in the Mulatos district, Sonora, Mexico, in Albinson, T., Nelson, C.E. (eds.), New Mines and Discoveries in Mexico and Central America: Society of Economic Geologists Special Publication Series, 8, 199-216.

Strujkov, S.F., Ryjov, O.B., Aristov, V.V., Grygoriev, N.V., Radchenko, Yu.I., Kolesnikov, A.G., Abbott, G.J., 1996, Geological structure and ore mineralogy of the Julietta gold-silver deposit, Northeast Russia: International Geology Reviews, 38, 625-648.

Thiersch, P.C., Williams-Jones, A.E., Clark, J.R., 1997, Epithermal mineralization and ore controls of the Shasta $\mathrm{Au}-\mathrm{Ag}$ deposit, Toodoggone District, British Columbia, Canada: Mineralium Deposita, 32, 44-57.

Tüysüz, N., Akçay, M., 1997, The gold occurrences in the volcanic arc of the eastern Pontides, NE-Turkey, in Papunen, H. (ed.), Mineral Deposits: Research and Exploration - where do they meet?: Rotterdam, Brookfield, A.A. Balkema, 331-334.

White, N.C., Hedenquist, J.W., 1990, Epithermal environments and styles of mineralization: variations and their causes, and guidelines for exploration, in Hedenquist, J.W., White, N.C., Siddeley, G. (eds.), Epithermal Gold Mineralization of the Circum-Pacific: Geology, Geochemistry, Origin and Exploration, II: Journal of Geochemical Exploration, 36, 445-474.

White, N.C., Leake, M.J., McCaughey, S.N., Parris, B.W., 1995, Epithermal gold deposits of the southwest Pacific: Journal of Geochemical Exploration, 54, 87-136.

Wood, D.G., Porter, R.G., White, N.C., 1990, Geological features of some Paleozoic epithermal gold occurrences in northeastern Queensland, Australia, in Hedenquist, J.W., White, N.C., Siddeley, G. (eds.), Epithermal Gold Mineralization of the Circum-Pacific: Geology, Geochemistry, Origin and Exploration, II: Journal of Geochemical Exploration, 36, 413-443.

Manuscrito recibido: Abril 30, 2003

Manuscrito corregido recibido: Julio 10, 2003

Manuscrito aceptado: Agosto 27, 2003 